Marquette University

e-Publications@Marquette

Electrical and Computer Engineering Faculty

Research and Publications

Engineering, College of

9-1-2016

Generalizing Effective Spatial Bandwidth for Pole Location in MAS: The Elliptic Cylinder

James Richie

Marquette University, james.richie@marquette.edu

Accepted version. IEEE Transactions on Antennas and Propagation, vol. 64, No. 9 (September 2016): 4123-4127. DOI. (C) 2016 IEEE. Used with permission. 


\section{Generalizing Effective Spatial Bandwidth for Pole Location in MAS: The Elliptic Cylinder}

\author{
James E. Richie, Senior Member, IEEE
}

\begin{abstract}
The location of poles in the Method of Auxiliary Sources for two-dimensional scatterers can be assisted using the effective spatial bandwidth (EBW) of the incident and scattered fields. Previously, only circular boundaries were considered. Here, EBW is extended to noncircular boundaries. Calculations presented for a cylinder with elliptical cross section verify EBW as an additional tool for pole placement for more general geometries.
\end{abstract}

Index Terms-Boundary value problems, Electromagnetic scattering, Electromagnetic theory

\section{INTRODUCTION}

The Method of Auxiliary Sources (MAS) [1] is a numerical procedure to compute the scattering of objects. MAS is one of several techniques that model the unknown fields using monopoles and/or multipoles at multiple origins. Other methods include the Multiple Multipole Program (MMP) [2], the filamentary current method (FCM) [3] and the Generalized Multipole Technique [4]. We shall refer to this collection of methods as "auxiliary source methods".

The main difficulty in using auxiliary sources at multiple origins is that the number, order, and location of the poles can have a large effect on the accuracy of the results. Some authors have developed rules to guide the placement of the poles for a particular problem. For example, [5] provides a set of guidelines that are later refined in [6]. The radius of curvature of the boundary can be useful for pole location as well. In [7], it is shown that the poles should become closer to the boundary and closer to each other as the radius of curvature decreases.

However, applying the rules for pole placement does not always provide a solution with acceptable accuracy. The use of randomly located poles in an iterative procedure was recently proposed [8], particularly for large scatterers where conventional methods may require a very large number of unknowns. In [8], the only restriction on the pole locations is that they may not be too close to the scatterer boundary.

To further investigate difficulties in applying the MAS method, some researchers have reported on the analysis of the MAS procedure applied to canonical problems. In [9], a study of the accuracy and sources of error in the MAS method is given for the problem of a circular cylindrical scatterer. In addition, the convergence of the fields and the pole strengths for the circular cylindrical scatterer are analysed in [10]. In fact, there are instances where the fields converge even though the pole strengths have large magnitudes and large phase variations.

In [11], the concept of Effective spatial BandWidth (EBW) is introduced and used to aid in the determination of pole locations for the case of a perfectly conducting circular cylindrical scatterer. The dielectric scatterer is discussed in [12].

EBW is a measure that indicates the amount of variation of a field component along a closed boundary. For example, the incident field in a scattering scenario has an EBW that indicates how much the incident field varies. Then, the scattered field model should have a similar EBW to obtain an accurate and stable solution. In auxiliary source methods, the scattered field model consists of poles. Therefore, pole locations can be chosen that provide a scattered field EBW along

J. Richie is with the Department of Electrical and Computer Engineering, Marquette University, Milwaukee, WI, 53233 USA e-mail: richiej@mu.edu.

Manuscript received ...; revised ... the boundary that is comparable to the incident field EBW. Thus, EBW can be used as an additional guide for pole placement in the auxiliary source methods.

In this paper, the computation of effective spatial bandwidth will be extended to two-dimensional scatterers with non-circular cross sections. After the fundamental procedures are discussed, they will be applied to a scatterer with elliptical cross section. It will be seen that the EBW calculations provide pole location information that is beneficial to the MAS solution of the scattering problem.

\section{THEORY}

In this section, we explain the modifications to EBW calculations that are used for more general boundary shapes. The theory for the circular case is provided in [11].

Consider a perfectly conducting scatterer that has uniform cross section in the $z$ direction, and an incident electric field with propagation vector in the $x-y$ plane and polarized in the $z$ direction. Then, the scattering problem can be reduced to a two-dimensional problem in the $z=0$ plane. Such scenarios are typically denoted two-dimensional $\mathrm{TM}^{z}$ problems.

Suppose that locations on the boundary, $\left(x_{b}, y_{b}\right)$, can be parameterized with $s \in(0, C)$, so that $x_{b}=f(s), y_{b}=g(s)$. Note that $s$ is a distance.

Let the incident electric field along the boundary of the scatterer be $E(s)$. This function is periodic and can be decomposed into an expansion of spatial frequencies that includes a fundamental term and higher order harmonics that are integer multiples of the fundamental.

The field, $E(s)$ can be limited in spatial bandwidth to a maximum harmonic of $N$ using the convolution:

$$
E_{N}(s)=\int_{\sigma=0}^{C} K_{N}(s, \sigma) E(\sigma) d \sigma
$$

where $N$ is the maximum spatial frequency in the bandlimited function $E_{N}(s)$, and

$$
K_{N}(s, \sigma)=\frac{1}{C} \frac{\sin \left[\left(N+\frac{1}{2}\right) \frac{2 \pi}{C}(s-\sigma)\right]}{\sin \left[\frac{1}{2} \frac{2 \pi}{C}(s-\sigma)\right]}
$$

where $C$ is the circumference of the scatterer boundary. The kernel, $K_{N}(s, \sigma)$ has two properties worth mentioning. First, it must be periodic over the boundary; second, when $N=0$, the kernel is constant so the integration returns the average value in the bandlimiting operation.

Define the energy of $E(s)$ as

$$
\mathcal{E}(E)=\int_{s=0}^{C}|E(s)|^{2} d s
$$

To estimate the EBW of $E(s)$, define the bandwidth as the spatial frequency range that contains $99.9 \%$ of the energy. That is, find the smallest $N$ so that

$$
\Delta_{N}=\frac{\mathcal{E}(E)-\mathcal{E}\left(E_{N}\right)}{\mathcal{E}(E)} \times 100 \%<0.1 \% .
$$

\section{EBW Results: Elliptical Cross Section}

A perfectly conducting cylindrical scatterer with elliptical cross section will be used to illustrate the generalization of EBW to noncircular cross sections. The EBW will be calculated for a plane wave incident field and for an internal monopole. Results are computed for a variety of elliptical geometries by numerically integrating (1) and (3). 


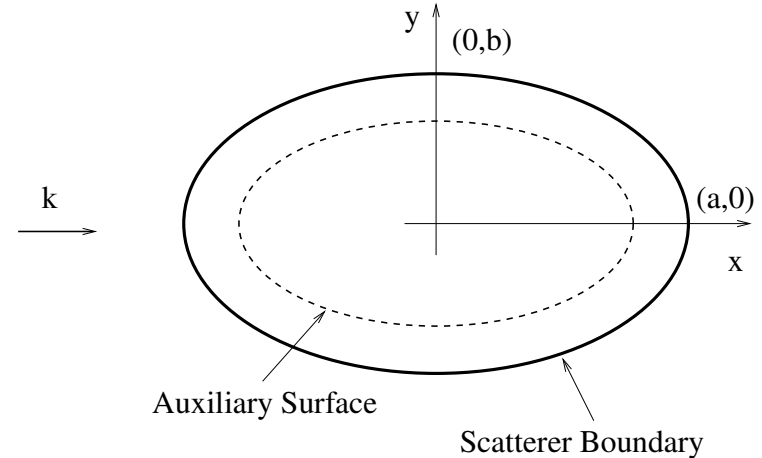

Fig. 1. Example geometry of scatterer and auxiliary surface used in MAS. The $\mathrm{k}$ vector as shown corresponds to an angle of incidence, $\alpha=0^{\circ}$.

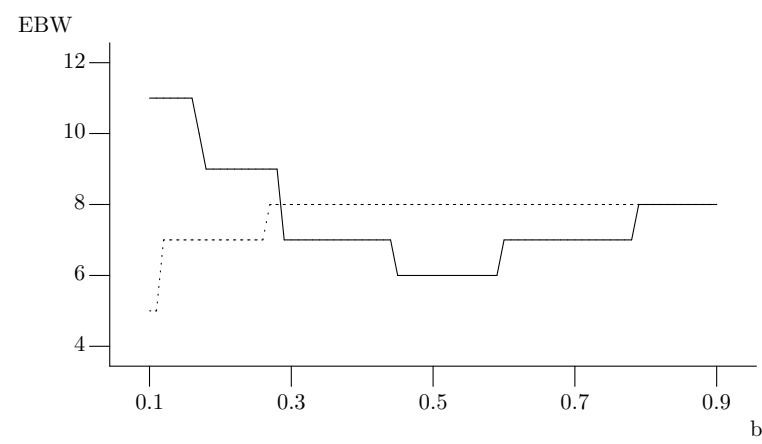

Fig. 2. EBW vs. $b$ for a plane wave incident on an elliptical cylinder with fixed $a=0.9 \lambda$. Solid: $\alpha=0^{\circ}$; Dotted: $\alpha=90^{\circ}$.

An elliptical cross section is shown in Fig. 1. The ellipse has a major axis along $x$ from $-a$ to $a$ and a minor axis along $y$ from $-b$ to $b$. Points along the ellipse can be found using

$$
x=a \cos (2 \pi t) \quad y=b \sin (2 \pi t)
$$

where $t$ is from 0 to 1 . An ellipse can also be characterized by its ellipticity, $e$ :

$$
e=\sqrt{\frac{a^{2}-b^{2}}{a^{2}}}
$$

where $e=0$ is a circle and $e=1$ is a line segment. The focal points for the ellipse are located at $\pm a e$.

\section{A. EBW for a Plane Wave Incident Field}

A $\mathrm{TM}^{z}$ plane wave incident field on the cylinder can be defined by its amplitude $E_{0}$, wavelength $(\lambda=2 \pi / k)$ and angle of incidence $(\alpha)$. Fig. 1 shows the angle of incidence at $180^{\circ}$. The electric field only has a $z$ component given by:

$$
E_{z}^{P W}=E_{o} e^{j k(x \cos \alpha+y \sin \alpha)}
$$

where an $e^{j \omega t}$ time dependence is assumed throughout. Note that the EBW for the plane wave will depend on the incident angle as well as the shape of the scatterer.

The EBW for a plane wave incident at $\alpha=0^{\circ}$ and $90^{\circ}$ on an ellipse with $a=0.9 \lambda$ vs. $b$ is shown in Fig. 2. A plane wave incident field has a constant magnitude, so the only variation is the phase. The phase of the incident field along the boundary for the $b=0.15 \lambda$ case is plotted in Fig. 3 for $\alpha=0^{\circ}$ and $90^{\circ}$.

Near $b=a=0.9 \lambda$ (the right side of Fig. 2), the EBW for the plane wave is the same for both incident angles because the ellipse is nearly circular. The EBW=8 result for a plane wave incident on a $0.9 \lambda$ circular cylinder matches the calculations reported in [11].

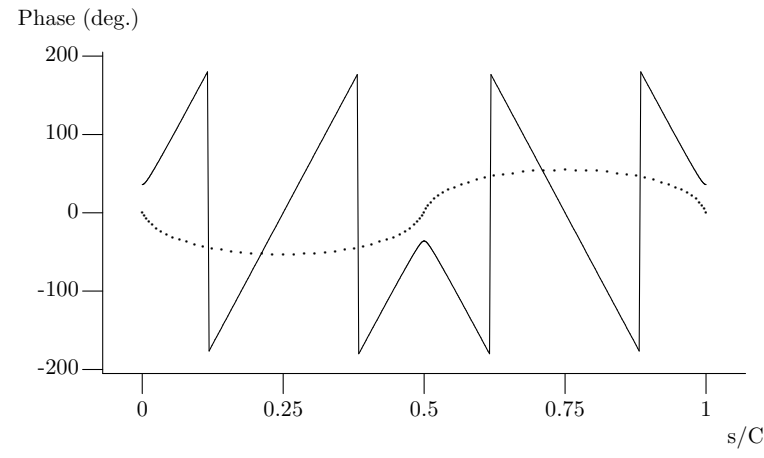

Fig. 3. Phase of plane wave incident field on an elliptic cylinder with $a=$ $0.9 \lambda, b=0.15 \lambda$. solid: $\alpha=0^{\circ}$; dotted: $\alpha=90^{\circ}$.

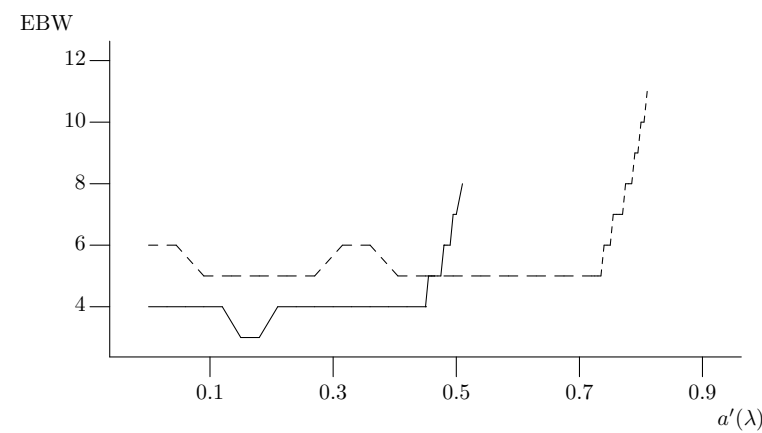

Fig. 4. EBW of an interior monopole at $\left(a^{\prime}, 0\right)$. Solid: $a=0.6 \lambda, b=0.4 \lambda$; dotted: $a=0.9 \lambda, b=0.5 \lambda$.

As $b$ decreases, the ellipse becomes thinner. For $\alpha=90^{\circ}$, the EBW stays constant until $b=0.3 \lambda$, and then falls. For a thin ellipse with $\alpha=90^{\circ}$, the wave fronts are parallel to the flatter portion of the ellipse, so the variation of the incident field is small as seen in Fig. 3. Thus, the EBW is smaller for small $b$.

For $\alpha=0^{\circ}$, the plane wave is perpendicular to the flatter portion of the ellipse. The EBW shown in Fig. 2 initially drops as $b$ decreases. As the ellipse becomes very thin, the EBW rises. The phase of the incident field for $b=0.15 \lambda$ at an incident angle of $0^{\circ}$ given in Fig. 3 shows the much larger variations in phase.

Calculations for other ellipse geometries provide similar results. At $\alpha=0^{\circ}$, the plane wave EBW often drops below the value for the circular case; however, at $\alpha=90^{\circ}$, the EBW typically does not change until the drop for very low $b$.

\section{B. EBW for an Internal Monopole}

The MAS model for the scattered field is a collection of monopoles inside the elliptical boundary. A monopole is a line source in the $z$ direction [13, Section 5.6]. The field at $\vec{\rho}$ of a unit strength monopole at $\vec{\rho}^{\prime}$ can be written as:

$$
E_{z}^{\text {mono }}=H_{0}^{(2)}\left(k\left|\vec{\rho}-\vec{\rho}^{\prime}\right|\right)
$$

where $k$ is the wavenumber, and $H_{0}^{(2)}(x)$ is the Hankel function of the second kind of order 0 representing outgoing waves.

The EBW for a monopole is also computed using the relations from Section II. The EBW for a monopole along the positive $x$ axis (at $a^{\prime}$ ) is shown in Fig. 4 for the ellipse cases $a=0.6 \lambda, b=0.4 \lambda$ and $a=0.9 \lambda, b=0.5 \lambda$.

The EBW for the monopole at small $a^{\prime}$ in both cases has some changes, similar to the small changes in the plane wave EBW case. More importantly, the EBW rises quickly as $a^{\prime}$ approaches $a$. At these locations, the EBW of the monopole is reaching the location 


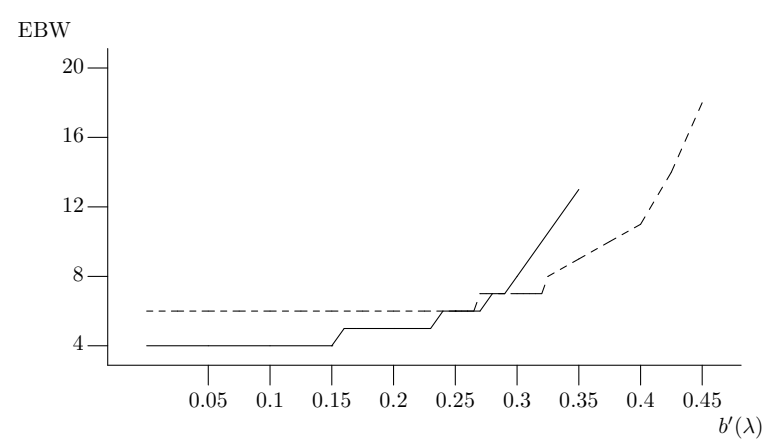

Fig. 5. EBW of an interior monopole at $\left(0, b^{\prime}\right)$. Solid: $a=0.6 \lambda, b=0.4 \lambda$; dotted: $a=0.9 \lambda, b=0.5 \lambda$.

where it matches and then exceeds the EBW of an incident plane wave.

The EBW for a monopole displaced along the $y$ axis (at $b^{\prime}$ ) using the same scatterer parameters is shown in Fig. 5. In the case of a ydisplaced monopole, the EBW begins small and eventually increases as $b^{\prime}$ increases. The monotonic increase has been consistently found for y-displaced monopoles and a variety of ellipse parameters.

\section{GMT/MAS RESULTS AND DISCUSSION}

In this section we shall review the MAS method, and then investigate simulation results with respect to the EBW data presented in the previous section.

\section{A. The Method of Auxiliary Sources (MAS)}

The solution for two-dimensional $\mathrm{TM}^{z}$ scattering for perfectly conducting scatterers consists of computing the scattered field. The MAS technique uses a model for the scattered field where lowestorder poles are placed along an auxiliary surface (AS) inside the scatterer, as shown in Fig. 1. The model for the scattered field is written as a sum of $M_{0}$ monopoles:

$$
E_{z}^{s}(\vec{\rho})=\sum_{m=1}^{M_{0}} a_{m} H_{0}^{(2)}\left(k\left|\vec{\rho}-\vec{\rho}_{m}^{\prime}\right|\right)
$$

where $a_{m}$ is the unknown amplitude of pole $m$ located at $\vec{\rho}_{m}^{\prime}$.

The $a_{m}$ coefficients are found by satisfying the boundary condition:

$$
E_{z}^{i}(\vec{\rho})+E_{z}^{s}(\vec{\rho})=0
$$

where $\vec{\rho}$ is on the scatterer boundary. Choosing $L=M_{0}$ specific points along the boundary $\left(\vec{\rho}_{\ell}, \ell=1,2, \ldots, L\right)$, the system of equations

$$
-E_{z}^{i}\left(\vec{\rho}_{\ell}\right)=\sum_{m=1}^{M_{0}} a_{m} H_{0}^{(2)}\left(k\left|\vec{\rho}_{\ell}-\vec{\rho}_{m}^{\prime}\right|\right)
$$

is solved to obtain $a_{m}$. The $\vec{\rho}_{\ell}$ locations are equally spaced in arc length around the boundary.

Once the solution is found, two measures will be used to evaluate the suitability of the result. The first is to look at the average percent error in the boundary condition, computed as:

$$
\bar{\varepsilon} \%=\frac{1}{P} \sum_{p=1}^{P} \frac{\left|E_{z}^{i}\left(s_{p}\right)+E_{z}^{s}\left(s_{p}\right)\right|}{\left|E_{z}^{i}\left(s_{p}\right)\right|} \times 100 \%
$$

where $s_{p}$ is a set of $P$ equally spaced points (in arc length) on the boundary and $P$ is much larger than $M_{0}$.

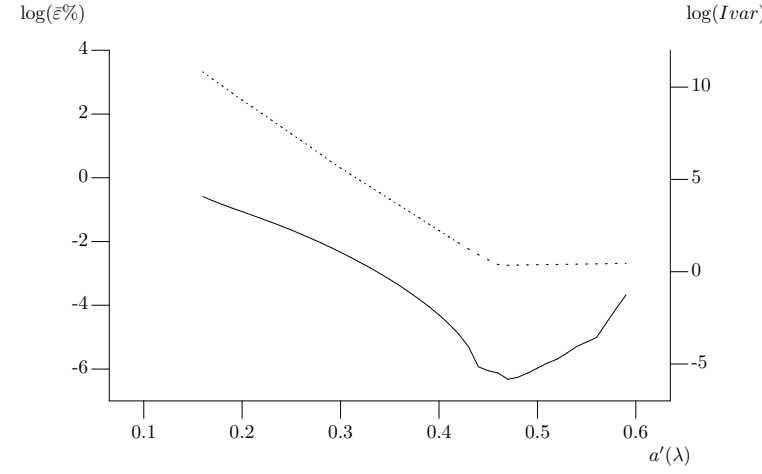

Fig. 6. MAS results plot of $\log (\bar{\varepsilon} \%)$ (solid) and $\log ($ Ivar $)$ (dotted) as a function of $a^{\prime}$ for an elliptical cross section with $a=0.6 \lambda$ and $b=0.4 \lambda$.

A second measure is related to the stability of the solution, or in other words, the convergence of the coefficients. In some cases, the MAS coefficients have exceedingly large values and nearly opposite phases [11]. Then, as $M_{0}$ increases, the coefficients tend to diverge. A measure was proposed in [11] to estimate the stability of the solution. The measure used in [11] will not be used here. In [14] a more appropriate measure is suggested:

$$
\text { Ivar }=\max \left\{\left|a_{n}\right|\right\}
$$

which is a simpler and equally informative measure of the suitability of the solution.

\section{B. EBW Verification via MAS Simulation}

In this section, the MAS method will be used to test the EBW results provided in Section III. The goal is to verify that EBW provides useful information regarding the location of the poles to obtain suitable solutions.

The MAS solution is also affected by the location of the poles relative to the singularities of the scattered field [4, Ch. 5]. In general, the auxiliary surface must enclose the singularities of the scattered field. For the elliptical cylinder considered, the line segment between the focal points is the locus of singularities of the scattered field.

Consider a cylindrical scatterer with elliptical cross section and dimensions $a=0.6 \lambda$ and $b=0.4 \lambda$. The plane wave is incident at $0^{\circ}$. The plane wave EBW is 5. An interior monopole has EBW=5 at $a^{\prime}=0.455 \lambda$ and at $b^{\prime}=0.16 \lambda$.

The location of the poles in the simulations that follow will be on an auxiliary surface that is an ellipse of size $\left(a^{\prime}, b^{\prime}\right)$. Fig. 6 shows $\bar{\varepsilon} \%$ and Ivar vs. $a^{\prime}$ for an elliptical cylinder scatterer with $a=0.6 \lambda$ and $b=0.4 \lambda$. The auxiliary surface has $b^{\prime}=0.16 \lambda$ and variable $a^{\prime}$. The number of poles used is 40 . At low $a^{\prime}$ values, Ivar is very large. This indicates that the coefficients are very large and do not represent a suitable solution. If the number of poles increases, Ivar increases as well. In short, the coefficients will diverge if $M_{o}$ increases.

As $a^{\prime}$ increases, Ivar decreases. The value of Ivar settles near 1 at $a^{\prime}=0.45 \lambda$. Since the plane wave EBW matches the interior monopole EBW at $a^{\prime}=0.455 \lambda$, we conclude that the EBW results have predicted the stable solution region indicated by the low Ivar.

The ellipticity $e$ of the boundary is 0.745 so that the focal distance is $a e=0.4472$. Therefore, the $a^{\prime}=0.45 \lambda$ EBW result is consistent with the requirement that the singularities be enclosed by the auxiliary surface. The Ivar results also confirm this requirement.

As $a^{\prime}$ increases, The boundary error decreases and then increases. As $a^{\prime}$ approaches $a$, the poles are quite far apart compared to the distance of each pole to the boundary. It is well known that the distance between neighboring poles should be on the order of the 
distance from the pole to the boundary. The increase in boundary error as $a^{\prime} \rightarrow a$ is expected because the poles are approaching the boundary and moving farther apart.

The minimum boundary condition error is $1.82 \times 10^{-6}$ and occurs at $a^{\prime}=0.47 \lambda$. The boundary condition error is below $10^{-5}$ for $a^{\prime}$ between 0.44 and $0.51 \lambda$. This range begins near the $a^{\prime}$ predicted from EBW (0.455 $\lambda)$.

\section{Discussion}

The EBW data for a variety of elliptical cross sections have been evaluated. In most cases, the EBW calculations provide an auxiliary surface that encloses the singularities, provides a low boundary condition error, and the coefficients are well behaved. However, for very thin elliptical cross sections, the EBW analysis does not lead to suitable MAS solutions. The breakdown for EBW results is estimated to occur for ellipticities of roughly 0.9 or larger.

Cases of high ellipticity are troublesome because EBW is a global property of the scattering problem. High ellipticity means that the scatterer effectively has a rounded wedge. The EBW calculation compares the energy of the initial boundary field to the band-limited boundary field. In the case of a corner, the total energy near the corner may be quite small and the EBW value required for the corner is not obtained. In such cases, local techniques for corners [1] can be used to obtain suitable solutions.

For example, consider an ellipse boundary with $a=0.6 \lambda$ and $b=0.225 \lambda$, which has a high ellipticity of 0.927 . The EBW for the $+x$-propagating plane wave is 6 . An interior monopole displaced along $x$ reaches $E B W=6$ near $x=0.425$. An interior monopole displaced along $y$ reaches $E B W=6$ near $y=0.1125$. Using $a^{\prime}=$ 0.425 and $b^{\prime}=0.1125$ with 40 poles results in $\bar{\varepsilon} \%=20,609$ with $I_{\text {var }}=124,730$. Note that the auxiliary surface does not enclose scattered field singularities at $x= \pm 0.555, y=0$.

To remedy this poor solution, we first introduce an overdetermined solution to the matrix equation by doubling the number of observation points, i.e., $L=2 M_{o}$ [5]. An overdetermined matrix solution tends to reduce the volatility of the solution and is not necessary if the ellipticity is smaller. In the example, using $L=2 M_{o}$ results in a boundary error $\bar{\varepsilon} \%=4.6 \%$ and $I_{\text {var }}=4.05$.

The error and $I_{v a r}$ values obtained may still be unacceptable. Locations of high curvature can be accounted for by placing additional poles near the high curvature locations. six additional monopoles are at the locations $x= \pm 0.578, y=0$ and $x= \pm 0.555, y= \pm 0.01$. For each additional monopole, two additional test points are added on the boundary near the high curvature locations. The error for this scenario is $\bar{\varepsilon} \%=0.4055 \%$ and $I_{v a r}=0.482$. These values can be considered acceptable in many cases.

EBW calculations provide additional insight into the location of poles for scattering problems that complements the rules provided in [6]. Combining the local rules of [6] with the global EBW calculations, a clearer picture of the best locations can be obtained. The largest benefit of EBW calculations is avoiding poorly behaved coefficients; this is demonstrated here using the Ivar measure and a low Ivar indicates a stable solution.

The stability of the MAS solution has been verified by increasing $M_{0}$. For cases where $\left(a^{\prime}, b^{\prime}\right)$ are chosen according to EBW results, simulations indicate that Ivar is very stable and $\bar{\varepsilon} \%$ decreases as $M_{0}$ increases.

Finally, it is important to note that the calculations used to estimate the EBW for the boundary fields is essentially a Fourier series calculation. Thus, the computations can be done quickly using wellknown Fourier methods and not add a heavy computational burden.

\section{CONCLUSION}

The EBW concept and calculations have been extended to noncircular geometries. As an example, the EBW calculations for scatterers with elliptical cross sections is shown to provide pole location information that results in accurate solutions with stable coefficients.

\section{ACKNOWLEDGMENT}

The author would like to thank the editors and reviewers for their helpful comments and suggestions.

\section{REFERENCES}

[1] D. I. Kaklamani and H. T. Anastassiu, "Aspects of the method of auxiliary sources (MAS) in computational electromagnetics," IEEE Antennas and Propagation Magazine, vol. 44, no. 3, pp. 48-64, Jun. 2002.

[2] C. Hafner, Post-Modern Electromagneitcs: Using Intelligent Maxwell Solvers. New York, NY: John Wiley \& Sons, 1999.

[3] Y. Leviatan and A. Boag, "Analysis of electromagnetic scattering from dielectric cylinders using a multifilament current model," IEEE Trans. Antennas Propag., vol. 35, pp. 1119-1127, Oct. 1987.

[4] T. Wriedt, Ed., Generalized Multipole Techniques for Electromagnetic and Light Scattering, ser. Computational Methods in Mechanics. New York, NY: Elsevier Science B. V., 1999, vol. 4.

[5] C. Hafner, The Generalized Multipole Technique for Computational Electromagnetics. Boston, MA: Artech House, 1990.

[6] E. Moreno, D. Erni, C. Hafner, and R. Vahldieck, "Multiple multipole method with automatic multipole setting applied to the simulation of surface plasmons in metallic nanostructures," J. Opt. Soc Am. A, vol. 19, no. 1, pp. 101-111, Jan. 2002.

[7] K. Beshir and J. E. Richie, "On the location and number of expansion centers for the generalized multipole technique," IEEE Trans. Electromagn. Compat., vol. 38, no. 2, pp. 177-180, May 1996.

[8] M. A. Moharram and A. A. Kishk, "Electromagnetic scattering from two-dimensional arbitrary objects using random auxiliary sources," IEEE Antennas and Propagation Magazine, vol. 57, no. 1, pp. 204-216, Feb. 2015.

[9] H. T. Anastassiu, D. G. Lymperopoulos, and D. I. Kaklamani, "Accuracy analysis and optimization of the method of auxiliary sources (MAS) for scattering by a circular cylinder," IEEE Trans. Antennas Propag., vol. 52, no. 6, pp. 1541-1547, Jun. 2004.

[10] G. Fikioris, "On two types of convergence in the method of auxiliary sources," IEEE Trans. Antennas Propag., vol. 54, no. 7, pp. 2022-2033, Jul. 2006.

[11] J. E. Richie, "MAS pole location and effective spatial bandwidth of the scattered field," IEEE Trans. Antennas Propag., vol. 58, no. 11, pp. 3610-3615, Nov. 2010.

[12] _ - "Application of spatial bandwidth concepts to MAS pole location for dielectric cylinders," IEEE Trans. Antennas Propag., vol. 59, no. 12, pp. 4861-4864, Dec. 2011.

[13] R. F. Harrington, Time-Harmonic Electromagnetic Fields. New York, NY: McGraw-Hill Book Company, 1961.

[14] C. A. Valagiannopoulos, N. L. Tsitsas, and G. Fikioris, "Convergence analysis and oscillations in the method of fictitious sources applied to dielectric scattering problems," J. Opt. Soc Am. A, vol. 29, no. 1, pp. 1-10, Jan. 2012. 